\title{
How conclusive is the CONCLUDE trial?
}

\author{
Stefano Del Prato ${ }^{1}$
}

Received: 11 December 2019 / Accepted: 16 December 2019 / Published online: 16 January 2020

(C) Springer-Verlag GmbH Germany, part of Springer Nature 2020

\begin{abstract}
The development of basal insulin analogues has reduced the risk of hypoglycaemia in insulin-treated individuals with type 2 diabetes. Insulin degludec and insulin glargine $300 \mathrm{U} / \mathrm{ml}$ (glargine U300) represent an evolution of basal insulin analogues, both of them reducing the risk of hypoglycaemia as compared with that associated with glargine U100. However, whether degludec and glargine U300 are equivalent with respect to glycaemic control and risk of hypoglycaemia remains to be fully ascertained. In the CONCLUDE trial, 1609 individuals with type 2 diabetes were randomised to either degludec $200 \mathrm{U} / \mathrm{ml}$ (degludec U200) or glargine U300. In this issue of Diabetologia (https://doi.org/10.1007/s00125-019-05080-9) the investigators report that during the maintenance period, $\mathrm{HbA}_{1 \mathrm{c}}$ improved to a similar extent in the two groups with no significant difference in the rate of overall hypoglycaemia (the primary endpoint of the study), while rates of nocturnal symptomatic and severe hypoglycaemia (secondary endpoints) were lower with degludec U200 than with glargine U300. These results, although of great interest to the clinician, need to be carefully interpreted as they cannot be considered as conclusive. First, the primary endpoint was not met and, therefore, analyses of secondary endpoints remain exploratory. Even assuming that degludec is superior to glargine in reducing the risk of hypoglycaemia, the mechanism(s) accounting for such an advantage remain elusive and potential differences in pharmacokinetics and pharmacodynamics difficult to appreciate because of methodological issues. The study design had to be amended because of lack of reliability of the glucometers initially used in the trial, particularly in the low blood glucose ranges, so the potential implications of these changes in the subsequent conduct of the trial cannot be excluded. Finally, comparison with the BRIGHT trial, the only other available head-to-head study, is complicated by differences between the two studies in the primary endpoint ( $\mathrm{HbA}_{1 \mathrm{c}}$ reduction vs reduction of the risk of hypoglycaemia), study population (insulin-experienced vs insulin-naive) and concomitant glucose-lowering medications. In spite of all this, CONCLUDE teaches us an important lesson regarding the need, particularly in the clinical setting, to monitor the reliability of the glucometers the diabetic individual uses to adjust his/her insulin dose. Insufficient precision or inappropriate use of the glucometer can easily offset any minute advantage a new insulin can offer with respect to glycaemic control and risk of hypoglycaemia.
\end{abstract}

Keywords Basal insulin analogues · Degludec · Glargine U300 · Glucometer · Hierarchical statistical analysis · Home blood glucose monitoring $\cdot$ Hypoglycaemia $\cdot$ Pharmacodynamics $\cdot$ Pharmacokinetics $\cdot$ Type 2 diabetes

\section{Abbreviations CONFIRM}

Degludec U200

EMA

Glargine U300

Stefano Del Prato

stefano.delprato@med.unipi.it

1 Department of Clinical \& Experimental Medicine, Section of Diabetes, University of Pisa, Nuovo Ospedale Santa Chiara, Via Paradisa, 2, 56124 Pisa, Italy

Clinical Outcome assessmeNt of the eFfectiveness of Insulin degludec in Real-life Medical practice

Insulin degludec $200 \mathrm{U} / \mathrm{ml}$

European Medicines Agency

Insulin glargine $300 \mathrm{U} / \mathrm{ml}$
Basal insulins are commonly used in individuals with type 2 diabetes who fail to achieve and maintain satisfactory glycaemic control with non-insulin glucose-lowering agents [1]. However, the initiation of basal insulin is often delayed because of several hurdles, in particular, the high risk of hypoglycaemia [2]. This risk was particularly apparent with NPH and ultralente insulins, as a result of inappropriate peaking of insulin concentration in the middle of the night after bedtime administration [3]. The development of longacting insulin analogues (glargine and detemir) has significantly reduced the risk of hypoglycaemic events, particularly nocturnal events [4], and improved the chance of ensuring stricter glycaemic control in the morning after overnight fasting. This was mainly due to flatter plasma insulin 
pharmacokinetics and pharmacodynamics, longer duration of action and improved day-to-day reproducibility of insulin effects [3]. To further improve these features, new insulin formulations have been developed: insulin degludec and insulin glargine $300 \mathrm{U} / \mathrm{ml}$ (glargine U300) [5]. Insulin degludec owes its long half-life to conjugation to a fatty acid, which allows binding to circulating albumin and an excess of zinc and phenol, while it is the greater concentration of glargine U300 that ensures slower dissolution of the subcutaneous insulin depot and prolongation of its activity [6]. These new basal insulins have been shown to reduce the risk of hypoglycaemia compared with that associated with insulin glargine [7, 8], but whether degludec and glargine U300 are equivalent with respect to glycaemic control and risk of hypoglycaemia remains to be fully ascertained. The results of the CONCLUDE trial are published in this issue of Diabetologia [9]. In this study, a total of 1609 type 2 diabetic individuals previously treated with basal insulin and oral glucose-lowering agents (with the exclusion of secretagogues) were randomised to receive either degludec $200 \mathrm{U} / \mathrm{ml}$ (degludec U200) or glargine U300. During the maintenance period, $\mathrm{HbA}_{1 \mathrm{c}}$ improved to a similar extent in the two groups with no significant difference in the rate of overall hypoglycaemia (the primary endpoint of the study), while rates of nocturnal symptomatic and severe hypoglycaemia (secondary endpoints) were lower with degludec U200 than with glargine U300. Can we CONCLUDE that insulin degludec may offer an opportunity to improve glycaemic control while exposing individuals to a lower risk of hypoglycaemia than insulin glargine U300? Answering this question requires some points of interest to be addressed.

\section{Point 1: primary vs secondary endpoints}

The primary endpoint of the CONCLUDE trial was the rate of overall symptomatic hypoglycaemic events during the maintenance period. The difference between the two treatments for this endpoint failed to reach statistical significance and, accordingly, the authors conclude that no significant harm is associated with the use of degludec U200. Secondary endpoints, which included the rate of nocturnal symptomatic hypoglycaemic events, the rate of severe hypoglycaemic events during the maintenance period and overall symptomatic, nocturnal symptomatic and severe hypoglycaemic events during the total treatment period, were all lower with degludec than with glargine U300. Interpretation of secondary endpoints when the primary endpoint is not statistically significant, is controversial. According to current interpretation if a hierarchical procedure is used, after the primary endpoint failed to be rejected, analyses of secondary endpoints become exploratory. The guidelines of the European Medicines Agency (EMA) state 'Secondary endpoints may provide additional clinical characterisation of treatment effects but are, by themselves, not sufficiently convincing to establish the main evidence in an application for a licence or for an additional labelling claim'. They also state 'Secondary endpoints may be related to secondary objectives that become the basis for an additional claim, once the primary objective has been established' [10].

Interpretation of $p$ values is currently a matter of discussion. Although a call has been made to ban statistical significance in favour of compatible effect sizes [11], this has been claimed to foster statistical confusion and generate problematic issues with data interpretation, while relying on predefined uniform statistical rules allows more reliable comparisons and conclusions [12]. Therefore, the only solid conclusion that can be drawn for the results of the CONCLUDE trial and its statistical analysis is that there is uncertainty about the true superiority of insulin degludec with respect to risk of hypoglycaemia compared with insulin glargine U300. Moreover, if there was no difference in overall symptomatic hypoglycaemic events but the nocturnal ones were lower with degludec, one could argue that the rate of diurnal hypoglycaemia had to be lower with glargine U300. Such speculation adds further uncertainty regarding the real advantages/disadvantages of the two basal insulin analogues.

\section{Point 2: how could insulin degludec reduce risk of hypoglycaemia?}

Interpretation of the results of a trial may go beyond statistics as it may depend on other evidence that may corroborate data interpretation. In this respect, differences in pharmacokinetics/ pharmacodynamics could account for the different risks of hypoglycaemia. As mentioned, degludec and glargine U300 have flatter and more stable steady-state pharmacokinetic and pharmacodynamic profiles [5], with insulin degludec being claimed to have lower day-to-day variability in the glucoselowering effect [13]. Of interest, these data were obtained with degludec U100, whereas degludec U200 was used in CONCLUDE. No formal comparison between this insulin strength and glargine U300 is currently available. Similar pharmacokinetic/pharmacodynamic properties for degludec U100 and U200 have been inferred from the reproducibility of the insulin serum profile after the subcutaneous injection of the two insulins [14]. True differences between degludec and glargine U300, however, are difficult to determine as studies performed using the same clamp technique have suggested that glargine U300 may provide, as compared with degludec U100, less fluctuating $24 \mathrm{~h}$ pharmacodynamics and a more even pharmacokinetic profile [15]. It is also worth noting that all pharmacokinetic/pharmacodynamic parameters were obtained in type 1 diabetic individuals to avoid the interference of residual endogenous insulin secretion. Therefore, these 
pharmacokinetic/pharmacodynamic estimates do not necessarily apply directly to individuals with type 2 diabetes who retain variable degrees of endogenous insulin secretion. This, along with several other potentially confounding factors (Table 1), calls for some caution in interpreting pharmacokinetic/pharmacodynamic data in individuals with type 2 diabetes.

In summary, how insulin might account for a lower risk of hypoglycaemia is uncertain and carefully designed mechanistic studies may be necessary to appreciate differences that could justify a lower rate of hypoglycaemia, if this is in fact the case, in individuals with type 2 diabetes.

\section{Point 3: how does CONCLUDE compare with similar studies?}

CONCLUDE is the second randomised clinical trial comparing degludec and glargine U300, the first one being the BRIGHT study [16]. The two studies come to similar conclusions as far as glycaemic control is concerned. $\mathrm{HbA}_{1 \mathrm{c}}$ was significantly reduced at the end of the two studies with no difference observed between the two insulins. The rate of hypoglycaemia in BRIGHT was comparable for glargine U300 and degludec during the entire study, although a lower rate was reported with glargine U300 during the titration period. Conversely, in CONCLUDE, the rate of symptomatic hypoglycaemia over the entire study was numerically lower with degludec [9]. However, there are major differences in the two trials that may render a direct comparison troublesome (Table 2). First, the two studies had a different primary endpoint: glycaemic control in BRIGHT, overall number of hypoglycaemic events in CONCLUDE. The study populations are not comparable as the BRIGHT study recruited insulin-naive individuals, while insulin-experienced type 2 diabetic individuals were enrolled in CONCLUDE. Accordingly, the CONCLUDE diabetic population included individuals with a longer duration of diabetes and lower kidney function. The risk of hypoglycaemia is not necessarily attributable to insulin treatment as concomitant glucose-lowering agents may also contribute. With respect to this, use of sulfonylureas or glucagon-like peptide 1 receptor agonists was not allowed in CONCLUDE while $66 \%$ of the BRIGHT participants received a sulfonylurea. All these elements should be kept in mind when interpreting (and comparing) the results of the two studies. To some extent the two trials could be seen as complementary since one is looking at the time of insulin initiation while the other explores the effect of switching from NPH, glargine U100 or detemir to newer basal insulin formulations. This also implies that the results of the two trials cannot be generalised to the entire diabetic population.

Assessment of effectiveness and safety in real-world studies may provide better information on the impact of the two insulins in a broader type 2 diabetes population. In the Clinical Outcome assessmeNt of the eFfectiveness of Insulin degludec in Real-life Medical practice (CONFIRM) study [17], data on 4056 subjects were analysed. After 180 days of follow-up, degludec was associated with a larger reduction in $\mathrm{HbA}_{1 \mathrm{c}}(-3.0 \mathrm{mmol} / \mathrm{mol}$ $[-0.27 \%] ; p=0.03)$ and greater reductions in the likelihood of hypoglycaemia (OR $0.64 ; p<0.01)$ compared with glargine U300. In the DELIVER Naive D real-world study [18], mean decreases in $\mathrm{HbA}_{1 \mathrm{c}}$ were comparable in the glargine $\mathrm{U} 300$ and degludec cohorts $(-18.3 \pm 24.4 \mathrm{mmol} / \mathrm{mol}$ vs $-17.3 \pm 24.0 \mathrm{mmol} /$ mol $[-1.67 \pm 2.22 \%$ vs $-1.58 \pm 2.20 \%] ; p=0.51$ ) with no difference in the incidence of hypoglycaemia. The likelihood of insulin discontinuation may reflect the degree of risk of hypoglycaemia and exert an impact on patients' quality of life, as well as on healthcare costs. The two studies have generated different results and, as expected, discontinuation was $27 \%$ less likely with degludec than with glargine U300 in the CONFIRM study, while there was no difference between the discontinuation rates $(29.2 \%$ vs $32.6 \%$ for glargine U300 vs degludec, respectively; $p=0.14$ ) in the DELIVER Naive D study. In summary, no final conclusion can be drawn with respect to differentiation between insulin degludec and glargine U300 from the results of randomised clinical trials and real-world studies.
Table 1 Factors potentially affecting assessment of insulin pharmacokinetics/ pharmacodynamics

\begin{tabular}{ll}
\hline Item & Implication \\
\hline Different primary endpoints & Different question answered \\
Bioavailability of insulin analogues & Difficult proper comparison \\
Different metabolic effect of insulin & Uncertain glucose infusion rate \\
Time of insulin administration & Different time-action profiles \\
Residual effect of prior insulin administration & Uncertain initial insulin effect \\
Assessment of 'real' insulin concentrations & Difficult insulin concentration matching \\
Use of smoothed vs unsmoothed raw data & Effect of the smoothing algorithm \\
Clamp methodology & Intrinsic limitations even in expert hands \\
Glucose clamping quality & Poor reproducibility \\
Type 1 diabetes & Applicability to type 2 diabetes \\
\hline
\end{tabular}


Table 2 Main characteristics of the study population in CONCLUDE and BRIGHT

\begin{tabular}{lll}
\hline Characteristic & CONCLUDE & BRIGHT \\
\hline Primary endpoint & Confirmed symptomatic hypoglycaemia & Change in $\mathrm{HbA}_{1 \mathrm{c}}$ \\
Study population & Insulin-experienced individuals & Insulin-naive individuals \\
Age, years & $63 \pm 10$ & $60 \pm 10$ \\
Baseline $\mathrm{HbA}_{1 \mathrm{c}}, \mathrm{mmol}_{\mathrm{mol}}$ & $59 \pm 10$ & $70 \pm 9$ \\
Baseline $\mathrm{HbA}_{1 \mathrm{c}}, \%$ & $7.6 \pm 1.0$ & $8.6 \pm 0.8$ \\
Baseline eGFR, ml min ${ }^{-1}[1.73 \mathrm{~m}]^{-2}$ & $79 \pm 20$ & $92 \pm 26$ \\
Diabetes duration, years & $15 \pm 8$ & $11 \pm 6$ \\
Concomitant glucose-lowering therapies & $\%)$ & \\
Metformin & 78 & 92 \\
DPP4i & 21 & 24 \\
SGLT2i & 19 & 13 \\
TZD & 4 & 5 \\
SU & 0 & 66 \\
GLP1RA & 0 & 12 \\
\hline
\end{tabular}

DPP4i, dipeptidyl peptidase 4 inhibitor; GLP1RA, GLP1 receptor agonist; SGLT2i, sodium-glucose cotransporter 2 inhibitor; SU, sulfonylurea; TZD, thiazolidinedione

Data are presented as mean $\pm \mathrm{SD}$ or $\%$

\section{Point 4: the impact of protocol amendment}

During the trial the CONCLUDE investigators identified some inconsistency between self-monitoring of blood glucose values measured by the participants and values measured in the laboratory. Moreover, the number of participants reporting blood glucose-confirmed hypoglycaemic events was unexpectedly low while pseudo-hypoglycaemic events $(>3.9 \mathrm{mmol} / \mathrm{l}$ with symptoms) were more frequent. Also, individual reports from patients and investigators showed inconsistency between the patients' own blood glucose meter and the one supplied in the trial. These observations suggested potential failures of the glycaemic data collection system. Accurate assessment of the blood glucose meters used in the study showed that they did not meet the accuracy requirements specified by the International Organization for Standardization (ISO) and the US Food and Drug Administration (FDA) [19]. In particular, the devices displayed falsely higher results in the hypoglycaemic range accounting for an unusual pattern of hypoglycaemic events [20]. The investigators and the sponsor should be commended for pinpointing the problem and for ensuring the continuation of the study while protecting the patients' safety and preserving the scientific integrity of the study. This was achieved by a protocol amendment that introduced a 16 week variable maintenance period followed by a 36 week maintenance period [21].

In spite of the faultless handling of this unfortunate situation by the investigators and trial's sponsor, the potential implications of changes to the study design in the subsequent conduct of the trial should be considered. Falsely elevated glucose readings were likely to trigger excessive insulin titration, with an unwanted increase in the number of hypoglycaemic events.
Recurrent hypoglycaemic episodes in the titration and early maintenance period might have altered counterregulatory symptom and cognitive function responses to subsequent hypoglycaemic events in the final maintenance period. Alternatively, the same excess of hypoglycaemic events could have made participants more cautious, leading them to aim for slightly higher blood glucose levels in the fasting state and rendering them more alert with respect to the risk of hypoglycaemia. All these elements may have interfered with the possibility of assessing the true risk of hypoglycaemia associated with degludec and glargine U300.

\section{Final reflections on the CONCLUDE trial}

The CONCLUDE trial was an ambitious and innovative trial as its endpoints were based on the risk of hypoglycaemia rather than the glucose-lowering efficacy. However, its results are not conclusive because the statistical interpretation does not support formal superiority of insulin degludec vs insulin glargine U300. Moreover, there is uncertainty about the mechanisms through which insulin degludec may reduce the risk of hypoglycaemia. The distribution of hypoglycaemic events between night and day remains to be fully explored. Finally, the generalisability of the results of CONCLUDE (and BRIGHT) is uncertain, and this uncertainty cannot be addressed by findings from currently available real-world studies.

In spite of all this, CONCLUDE can teach us a useful clinical lesson. As already mentioned, the investigators must be commended for recognising the poor performance of the glucose meter initially used in the trial. The importance of 
careful surveillance of blood glucose monitoring systems for safe and reliable estimates of glucose control in clinical trials has been recently emphasised [22]. However, if this is critical in a trial, unreliable glucose monitoring systems become a matter of major concern in the real-world clinical setting. Recent publications report that the accuracy of different meters can vary widely [23], with mean absolute relative differences (MARDs), the most common metric used to assess the performance of these devices, ranging from $5.6 \%$ to $20.8 \%$ [24]. Moreover, accuracy tends to be lower within the hypoglycaemic range [24]. Such variability coupled with low adherence to self-management of the disease [25] and the well-known relationship between frequency of selfmonitoring blood glucose and attainment of $\mathrm{HbA}_{1 \mathrm{c}}$ target values [26] can easily offset the advantages of any new insulin formulation both in terms of glucose-lowering efficacy and reduction of the risk of hypoglycaemia. Therefore, the clinician must always keep in mind that the translation of any potential benefit of new insulin analogues in clinical practice cannot just rely on the demonstration of superior efficacy and/ or safety but rather it requires careful integration in the patient's self-management and education as well as continuous surveillance of glucose meters and their proper utilisation.

Acknowledgements I am indebted to the EASD for giving me the task of delivering an independent commentary on the occasion of the presentation of the results of the CONCLUDE trial at the 55th Annual Meeting of the EASD. This paper is largely based on that commentary. I am also grateful to G. Bolli (University of Perugia, Perugia, Italy), T. Heise (Profil, Neuss, Germany), J. Rosenstock (Dallas Diabetes Research Center at Medical City, Dallas, TX, USA) and the CONCLUDE trial investigators A. Philis-Tsimikas (Scripps Whittier Diabetes Institute, San Diego, CA, USA) and T. R. Pieber (Division of Endocrinology and Diabetology, Department of Internal Medicine, Medical University of Graz, Graz, Austria) for constructive discussion and for sharing their views, some of which I have reported in the paper.

Funding This work was funded by the University of Pisa, Department of Clinical and Experimental Medicine.

Duality of interest The author has received research funding from AstraZeneca, Boehringer Ingelheim, Novartis Pharmaceuticals Co. and Merck Sharpe \& Dohme; and is a consultant for or has received honoraria from AstraZeneca, Boehringer Ingelheim, Eli Lilly and Company, GlaxoSmithKline, Janssen Pharmaceuticals, Laboratoires Servier, Merck Sharp \& Dohme, Novartis Pharmaceuticals Co., Novo Nordisk, Sanofi and Takeda.

Contribution statement The author is the sole contributor to this paper.

\section{References}

1. Davies MJ, D'Alessio DA, Fradkin J et al (2018) Management of hyperglycaemia in type 2 diabetes, 2018. A consensus report by the American Diabetes Association (ADA) and the European Association for the Study of Diabetes (EASD). Diabetologia 61(12):2461-2498. https://doi.org/10.1007/s00125-018-4729-5
2. Russell-Jones D, Pouwer F, Khunti K (2018) Identification of barriers to insulin therapy and approaches to overcoming them. Diabetes Obes Metab 20(3):488-496. https://doi.org/10.1111/ dom. 13132

3. Peterson GE (2006) Intermediate and long-acting insulins: a review of NPH insulin, insulin glargine and insulin detemir. Curr Med Res Opin 22(12):2613-2619. https://doi.org/10.1185/030079906X154178

4. Monami M, Marchionni N, Mannucci E (2008) Long-acting insulin analogues versus NPH human insulin in type 2 diabetes: a metaanalysis. Diabetes Res Clin Pract 81(2):184-189. https://doi.org/10. 1016/j.diabres.2008.04.007

5. Goldman J, Kapitza C, Pettus J, Heise T (2017) Understanding how pharmacokinetic and pharmacodynamic differences of basal analog insulins influence clinical practice. Curr Med Res Opin 33(10): 1821-1831

6. Cahn A, Miccoli R, Dardano A, Del Prato S (2015) New forms of insulin and insulin therapies for the treatment of type 2 diabetes. Lancet Diabetes Endocrinol 3(8):638-652. https://doi.org/10.1016/ S2213-8587(15)00097-2

7. Roussel R, d'Emden MC, Fisher M et al (2018) Glycaemic control and hypoglycaemia in people with type 2 diabetes switching from twice-daily basal insulin to once-daily insulin glargine $300 \mathrm{U} / \mathrm{mL}$ or insulin glargine $100 \mathrm{U} / \mathrm{mL}$ (EDITION 1 and EDITION 2 subgroup analysis). Diabetes Obes Metab 20(2):448-452

8. Russell-Jones D, Gall MA, Niemeyer M, Diamant M, Del Prato S (2015) Insulin degludec results in lower rates of nocturnal hypoglycaemia and fasting plasma glucose vs. insulin glargine: a meta-analysis of seven clinical trials. Nutr Metab Cardiovasc Dis 25(10):898-905. https://doi.org/10.1016/j.numecd.2015.06.005

9. Philis-Tsimikas A, Klonoff DC, Khunti K et al (2020) Risk of hypoglycaemia with degludec versus glargine U300 in insulintreated patients with type 2 diabetes: the randomised, head-tohead CONCLUDE trial. Diabetologia. https://doi.org/10.1007/ s00125-019-05080-9

10. European Medicines Agency. Guideline on multiplicity issues in clinical trials. 2016. Available from www.ema.europa.eu/en/ documents/scientific-guideline/draft-guideline-multiplicity-issuesclinical-trials_en.pdf. Accessed 15 November 2019

11. Amrhein V, Greenland S, McShane B (2019) Scientists rise up against statistical significance. Nature 567(7748):305-307

12. Ioannidis JPA (2019) The importance of predefined rules and prespecified statistical analyses. Do not abandon significance. JAMA 312(21):2067-2068

13. Heise T, Nørskov M, Nosek L, Kaplan K, Famulla S, Haahr HL (2017) Insulin degludec: lower day-to-day and within-day variability in pharmacodynamic response compared with insulin glargine $300 \mathrm{U} / \mathrm{mL}$ in type 1 diabetes. Diabetes Obes Metab 19(7):10321039. https://doi.org/10.1111/dom. 12938

14. Korsatko S, Deller S, Koehler G et al (2013) A comparison of the steady-state pharmacokinetic and pharmacodynamic profiles of 100 and $200 \mathrm{U} / \mathrm{mL}$ formulations of ultra-long-acting insulin degludec. Clin Drug Investig 33(7):515-521. https://doi.org/10.1007/s40261013-0096-7

15. Bailey TS, Pettus J, Roussel R et al (2018) Morning administration of $0.4 \mathrm{U} / \mathrm{kg} /$ day insulin glargine $300 \mathrm{U} / \mathrm{mL}$ provides less fluctuating 24-hour pharmacodynamics and more even pharmacokinetic profiles compared with insulin degludec $100 \mathrm{U} / \mathrm{mL}$ in type 1 diabetes. Diabetes Metab 44(1):15-21. https://doi.org/10.1016/j.diabet. 2017.10.001

16. Rosenstock J, Cheng A, Ritzel R et al (2018) More similarities than differences testing insulin glargine 300 units $/ \mathrm{mL}$ versus insulin degludec 100 units $/ \mathrm{mL}$ in insulin-naive type 2 diabetes: the randomized head-to-head BRIGHT trial. Diabetes Care 41(10): 2147-2154. https://doi.org/10.2337/dc18-0559

17. Tibaldi J, Hadley-Brown M, Liebl A et al (2019) A comparative effectiveness study of degludec and insulin glargine $300 \mathrm{U} / \mathrm{mL}$ in 
insulin-naïve patients with type 2 diabetes. Diabetes Obes Metab 21:1001-1009

18. Sullivan SD, Nicholls CJ, Gupta RA et al (2019) Comparable glycaemic control and hypoglycaemia in adults with type 2 diabetes after initiating insulin glargine 300 units $/ \mathrm{mL}$ or insulin degludec: the DELIVER Naïve D real-world study. Diabetes Obes Metab 21(9):2123-2132. https://doi.org/10.1111/dom.13793

19. Demircik F, Kirsch V, Ramljak S, Vogg M, Pfützner AH, Pfützner A (2019) Laboratory evaluation of linearity, repeatability, and hematocrit interference with an internet-enabled blood glucose meter. J Diabetes Sci Technol 13(3):514-521

20. Pfützner A, Demircik F, Kirsch V et al (2019) System accuracy assessment of a blood glucose meter with wireless internet access associated with unusual hypoglycemia patterns in clinical trials. J Diabetes Sci Technol 13(3):507-513. https://doi.org/10.1177/ 1932296819841353

21. Philis-Tsimikas A, Stratton I, Nørgård Troelsen L, Anker Bak B, Leiter LA (2019) Efficacy and safety of degludec compared to glargine 300 units $/ \mathrm{ml}$ in insulin-experienced patients with type 2 diabetes: trial protocol amendment (NCT03078478). J Diabetes Sci Technol 13(3):498-506

22. Klonoff DC (2019) Postmarket surveillance of blood glucose monitor systems is needed for safety of subjects and accurate determination of effectiveness in clinical trials of diabetes drugs and devices. J Diabetes Sci Technol 13(3):419-423

23. Klonoff DC, Parkes JL, Kovatchev BP et al (2018) Investigation of the accuracy of 18 marketed blood glucose monitors. Diabetes Care 41(8):1681-1688

24. Ekhlaspour L, Mondesir D, Lautsch N et al (2017) Comparative accuracy of 17 point-of-care glucose meters. J Diabetes Sci Technol 11(3):558-566. https://doi.org/10.1177/1932296816672237

25. Peyrot M, Rubin RR, Lauritzen T et al (2005) Psychosocial problems and barriers to improved diabetes management: results of the Cross-National Diabetes Attitudes, Wishes and Needs (DAWN) study. Diabet Med 22(10):1379-1385. https://doi.org/10.1111/j. 1464-5491.2005.01644.x

26. Bosi E, Scavini M, Ceriello A et al (2013) Intensive structured selfmonitoring of blood glucose and glycemic control in noninsulintreated type 2 diabetes: the PRISMA randomized trial. Diabetes Care 36(10):2887-2894. https://doi.org/10.2337/dc13-0092

Publisher's note Springer Nature remains neutral with regard to jurisdictional claims in published maps and institutional affiliations. 